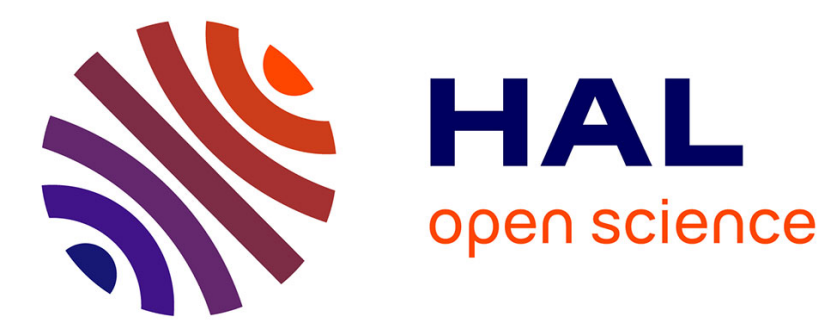

\title{
L'expérience de la crue. Comment redonner sens à son lieu de vie
}

\author{
François Duchêne, Christelle Morel Journel
}

\section{To cite this version:}

François Duchêne, Christelle Morel Journel. L'expérience de la crue. Comment redonner sens à son lieu de vie. Les Annales de la Recherche Urbaine, 2004. hal-01783657

\section{HAL Id: hal-01783657 \\ https://hal.science/hal-01783657}

Submitted on 2 May 2018

HAL is a multi-disciplinary open access archive for the deposit and dissemination of scientific research documents, whether they are published or not. The documents may come from teaching and research institutions in France or abroad, or from public or private research centers.
L'archive ouverte pluridisciplinaire HAL, est destinée au dépôt et à la diffusion de documents scientifiques de niveau recherche, publiés ou non, émanant des établissements d'enseignement et de recherche français ou étrangers, des laboratoires publics ou privés. 


\section{François Duchêne, Christelle Morel Journel L'EXPÉRIENCE DE LA CRUE}

$\mathbf{L}$ e risque naturel est défini de plus en plus couramment, par les gestionnaires du risque et par une part importante de la communauté scientifique, comme le point de rencontre d'un aléa et d'une vulnérabilitét. Derrière cette définition, largement consensuelle, tend à s'organiser une forme de plus en plus visible de division des tâches : aux Sciences de l'ingénieur, appliquées ou non, l'étude de l'aléa, et aux Sciences humaines et sociales, ou autres domaines d'application s'y rattachant, celle de la vulnérabilité. Dans ce second volet, la place réservée à l'étude des formes d'actions, matérielles ou idéelles, des «gens ordinaires », qualifiés ainsi de façon globalisante et générique face aux risques et à la catastrophe, reste marginale. Cette marginalisation trouve assez largement écho dans les politiques publiques touchant à la gestion des risques, tant il est vrai que «s'agissant de l'émergence de la "société civile", la référence à l'usager, si elle est affichée par l'État comme un élément de sa modernisation, tient aussi du discours incantatoire» (Reigner H., 2002, p. I6]. Or «pour que le peuple accepte d'être silencieux et sans voix [face à l'état du savoir], il aura fallu une extraordinaire et féconde invention» (Callon M. et alii, 200I, p. I7I). Cette approche excluante de la question du risque résulte d'une longue construction sociale. On peut se demander si l'édifice ainsi bâti ne présente pas quelques failles sitôt que l'on aborde la question au plus près des populations $a$ priori directement concernées par un danger.

Dans le droit fil de ce qui vient d'être énoncé, nous observons que la légitimité des procédures d'administration et d'interventions techniques autour des rivières repose, non sur des situations ordinaires et quotidiennes, mais davantage sur des moments de crise, en particulier lorsque les cours d'eau débordent, lorsque l'aléa prend corps. Cette légitimité s'exprime pour l'essentiel dans un dispositif conçu par et pour les gestionnaires du risque, dans une logique d'action qui, pour être efficace, se devrait de «gérer» la vulnérabilité, et pour ce faire, de considérer la population directement concernée, au mieux comme passive, et au pire comme gênante (Duchêne F., Morel Journel
Ch., 2000). Or, d'une part, après la crise et une fois éteints les projecteurs médiatiques, le «territoire» continue de travailler en profondeur (Langumier J., 2002). D'autre part, à l'écoute de riverains de cours d'eau débordants, on constate que cette activité est permanente et qu'elle se joue de frontières artificielles construites entre aléa et vulnérabilité.

Pour tenter de circonscrire les contours de cette activité, tant matérielle qu'idéelle, nous puiserons dans les résultats d'une enquête réalisée récemment auprès de riverains de l'Yzeron et du Ravin². À l'ouest de l'agglomération lyonnaise, l'Yzeron³ a un régime pluvial, caractérisé par des étiages sévères durant les périodes sans pluie et des débordements brusques suite à des orages violents. Et depuis la fin des années quatrevingt, ces crues sont apparues de plus en plus fréquentes (1983, I989, I990, I992, et 1993). Plus modeste, le bassin versant du Ravin est situé au nordest de l'agglomération lyonnaise. Le parcours accidenté de son lit provoque des écoulements très rapides, particulièrement violents et destructeurs en cas de crue d'orage. Pour autant l'histoire récente de ce site (depuis l'après-guerre) ne semble pas retenir de crues remarquables à l'exception de deux «événements », en I973 et I979, et d'inondations ponctuelles à l'occasion d'importants orages (en septembre I994 en particulier). Nous avons recueilli la parole d'habitants de zones identifiées comme étant inondables dans les Plans de prévention des risques (PPR) qui régissent ces deux cours d'eau. Notre choix méthodologique nous amène à ne pas privilégier ni introduire nous-mêmes la notion de «risque» lors de nos

I. Voir, par exemple, Dauphiné A., 200I, p. I7 ; ou Dubois-Maury J., 200I, p. 5 .

2. Pour plus de précision, voir Duchêne F., Morel Journel Ch., Coanus Th., Martinais E., 2003.

3. L'Yzeron, le plus long, se jette dans le Rhône après avoir parcouru $28 \mathrm{~km}$.

Les Annales de la recherche urbaine n 95, 0180-930-VI-04/95/pp. 71-77 ( 
rencontres. Pour autant, les crues et les inondations constituent bien des moments forts des entretiens 4 .

\section{Quand la boîte de Pandore s'entrouvre}

Au cours de notre enquête, nous avons progressivement pris conscience qu'une crue n'est pas nécessairement un problème dans l'absolu. La crainte de la crue débordante n'existe que relativement à un environnement, à une situation ou à un contexte particulier, d'ordre individuel et/ou collectif. Lorsque l'inondation survient, pour la victime il s'agit d'un événement marquant. Ce traumatisme intègre le chevauchement de dimensions individuelles et collectives et donne souvent lieu à des solidarités ponctuelles ou plus durables, qui vont marquer l'histoire générationnelle d'un territoire donné.

\section{Avant l'inondation, la rivière n'existe pas}

Ne pas avoir vécu la crue n'implique pas forcément n'en avoir aucune idée et/ou appréhension. Pour les riverains qui n'ont pas été confrontés aux crues de la rivière voisine, l'évocation de ces événements passés et possibles se construit en référence aux récits entendus, aux informations éparses glanées de ci de là et aux représentations du «malheur» qui intègrent histoires individuelles et collectives. Dans cette évocation, c'est souvent une tonalité abstraite qui domine, comme si l'accumulation de données «objectives» mathématiques, techniques, administratives, chronologiques, etc. - permettait d'approcher le phénomène sans le circonscrire tout à fait. Au bord de l'Yzeron par exemple, un riverain, nouvellement installé, aborde le problème des crues de manière extrêmement ordonnée, presque administrative. Il mentionne les deux dernières crues, les acteurs en charge du «dossier» et les décisions prises. Il restitue en quelque sorte la «mise sur agenda» de la question des crues, témoignant par là même de son traitement en cours qui justifie l'achat récent de sa maison :

«En gros, il y a eu plusieurs inondations, la dernière remonte à I993. Sinon, l'autre était avant I989. Depuis celle de I993, les eaux étaient montées pratiquement jusqu'à l'entreprise $\mathrm{X}$, et je crois qu'a été prise la décision de la part des diverses municipalités bordant l'Yzeron, d'entamer quelques travaux, pour justement essayer d'endiguer ce problème de débordement régulier, compte tenu qu'il y a quand même pas mal de maisons disséminées tout au long de l'Yzeron. Je crois que les travaux, d'après le voisin d'à côté, devraient débuter courant 2000, par l'aménagement de l'Yzeron, pour éviter que se renouvelle le problème qu'il y avait eu en I993.» (M. B., 40 ans, propriétaire, riverain de l'Yzeron depuis un mois).

Il n'est pas question d'incrédulité ici, mais d'une réaction raisonnable devant un problème que l'on peut appréhender de manière non moins raisonnable, puisque les autorités compétentes s'en occupent déjà. $\mathrm{Au}$ bord de l'Yzeron, les discours sont précis et circonstanciés parce que les riverains, et notamment ceux qui n'ont pas connu d'inondation, ont affaire avec l'existence avérée du phénomène et des dangers qui l'accompagnent pour les biens, voire les personnes. Pour le Ravin, la situation n'est pas la même puisque les inondations sont beaucoup plus anciennes et leur réalité parfois sujette à caution.

Pour certains riverains, la rivière ne vient à l'existence qu'après l'un de ses débordements, qui restera à jamais qualifié de premier. La «découverte» de la rivière pour des personnes qui l'ont quotidiennement sous les yeux découle de sa manifestation, que ce soit par le biais de débordements plus ou moins importants ou par des éléments déclenchant l'anticipation du risque d'inondation, telles les procédures de PPR par exemple. Mais, tant que cette manifestation n'a pas eu lieu, la rivière peut être facilement écartée du cadre de vie ou de la sphère des préoccupations. La crue, ou tout au moins son expérience par les riverains, transforme le rapport à la rivière, voire la rivière elle-même. D'ailleurs, une distinction est fréquemment introduite dans les propos recueillis entre «crue» et «débordement». Le second n'est pas considéré comme dangereux, et tout se passe comme s'il faisait encore partie du ruisseau, alors que la crue appartient clairement à un autre ordre de réalité. La référence fréquente aux traces physiques de l'inondation montre le potentiel de transformation de la rivière. Ces traces ${ }^{5}$, évidentes ou estompées, voire visibles seulement aux yeux de celui qui sait, sont devenues des signes de l'événement. Ces marques, si elles font aujourd'hui partie consubstantielle de la maison, de ce qu'elle contient, sont aussi des signes que les riverains peuvent mobiliser contre leur propre incrédulité et surtout pour convaincre les visiteurs, dont nous sommes, qu'ils n'ont pas rêvé. Cette dimension de preuve est encore plus forte lorsque des indemnisations sont en jeu.

Avec la crue, particulièrement soudaine pour ces cours d'eau à régime torrentiel, la rivière se transforme. Elle devient parfois méconnaissable et le grondement des flots sert souvent d'illustration à la «force de l'eau». En fait, non seulement il faut avoir vu pour

4. L'enquête s'est déroulée entre les mois de juillet et de novembre I999. Elle a porté auprès de 59 personnes au total, représentant 47 foyers, certains entretiens s'étant déroulés avec les deux membres d'un couple composant le même foyer. Toutes les personnes rencontrées vivent ou travaillent dans des zones identifiées à risques maximal (zone rouge) ou minimal (zone bleue) dans le Plan d'occupation des sols de leur commune. D'une durée d'une heure en moyenne, ces entretiens ont été intégralement retranscrits.

5. Terme dont le sens véhicule les idées d'«empreinte» et d'«égratignure». Cf. Rey A. (dir.), (I998), Le Robert. Dictionnaire historique de la langue française, p. 3872. 
croire à la possibilité d'inondations et à leur violence, mais il faut également avoir entendu. L'ouie joue un rôle fondamental dans la perception de la transformation de l'environnement. Une fois repérés, les signes de transformation de la rivière en crue servent d'éléments d'alerte pour les riverains : le bruit réveille, la force du courant impressionne. La couleur de l'eau compte aussi parmi ces repères. La gamme normale des couleurs du ruisseau calme s'oppose à celle du ruisseau en crue. La description des changements de la rivière est donc souvent une manière «objective » d'approcher la crue et ce qu'elle suscite pour les riverains. Bien souvent, l'appréhension diurne se transforme en angoisse la nuit. L'une des personnes rencontrées se rappelle que la famille n'a consenti à aller se coucher qu'une fois vérifiée la stabilité du niveau d'eau, comme si la position couchée et le sommeil rendaient encore plus vulnérable. L'imaginaire nocturne sied d'ailleurs si bien aux catastrophes que pour certains, elles paraissent n'arriver que dans l'obscurité. La transformation de la rivière rend l'eau lourde, sale, noire - terrible en un mot. L'étymologie des différents termes mobilisés ici renvoie à la saleté, à l'impureté, à la souillure dont les riverains craignent d'être atteints au travers de leur maison ou de ce qu'ils ont de plus précieux. Les rats qui arrivent aussi par le ruisseau parachèvent le tableau. Pour le Ravin, la question de la saleté s'avère cruciale. Le ruisseau passe en effet à proximité d'une décharge et les rats circulent dans la canalisation. La mention de leur présence traduit incontestablement le sentiment d'insécurité ressenti. Cette souillure, cette pollution évoquent aussi probablement quelque chose d'un ordre social rompu (Douglas M., 200I, p. 25].

\section{Le tissage des récits de crue}

À propos de riverains d'installations nucléaires, Françoise Zonabend notait toute l'importance de la parole dans le fait de (ne pas) dire le risque, «support de mille ruses destinées à obscurcir le sens déclaré, proclamé, de la narration entendue. Le langage ici peut dire ou taire, mener ou égarer, éclairer ou brouiller» (Zonabend F., I989, p. I4). Lors de notre enquête, l'évocation des émotions liées à la crue se mêle et s'entremêle aux récits d'inondations, ressurgit parfois dans le cadre de comparaisons, de rapprochements et d'autres associations discursives. Dans l'ensemble des propos recueillis, les récits de crue ne constituent pas forcément un moment isolé de l'entretien, ce qui fait que le terme «récit» s'avère finalement mal adapté. La forme la plus souvent rencontrée rappelle plutôt la technique du tissage, les évocations des crues formant la trame d'un récit dont les chaînes empruntent souvent à des logiques sociales, plus globales, en termes de position ou d'itinéraire. Dans certains entretiens, les éléments concernant les crues sont plus denses, mais ces passages

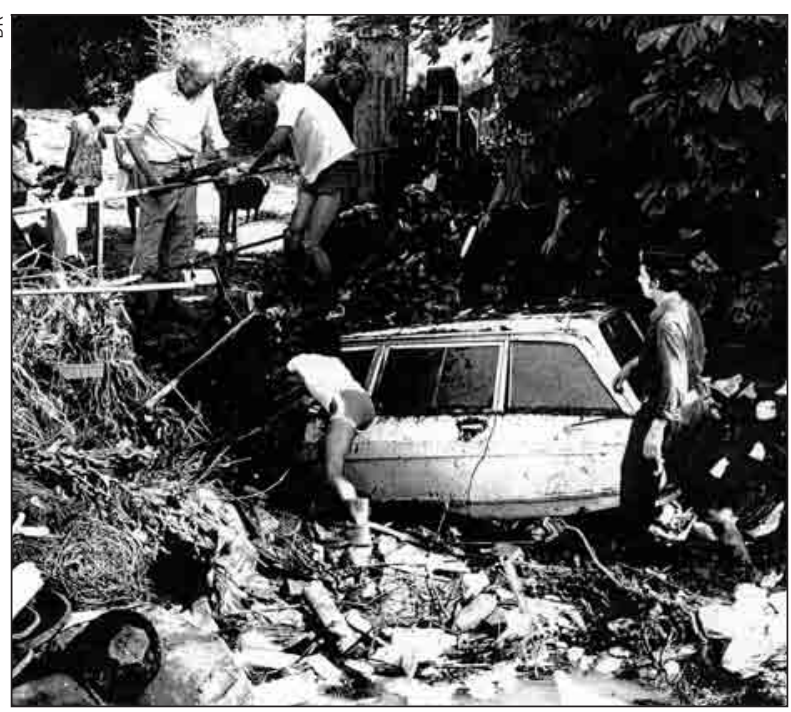

Un véhicule bloque le passage de l'eau en aval des serres Morel (crue du 11 août 1973).

n'en sont pas moins émaillés d'autres références qui leur confèrent une forme complexe. Parfois, tout se passe comme si l'on pouvait établir un lien entre la forme du récit de crue et ce qu'elle a suscité comme émotion. Des phrases courtes, une construction syncopée illustrent, par exemple, la soudaineté de la crue et l'inquiétude suscitée par le phénomène.

D'une manière générale, nous avons noté que, quand les riverains ont connu plusieurs crues ou débordements, les observations s'affinent au fur et à mesure des modifications de la configuration de la rivière. Que les personnes rencontrées choisissent de considérer la crue comme un phénomène total, en évoquant les différentes modifications du milieu ainsi que leurs conséquences sociales voire affectives, ou qu'elles ne conservent qu'un élément qui les a touchées de près et qui est censé traduire cet ensemble, elles ont engrangé quantité de données ou d'informations sur le cours d'eau et ses variations d'état. Ces données sont parfois «activées » pour anticiper et prévenir la prochaine crue.

Un riverain depuis plus de trente ans de l'Yzeron raconte ainsi la crue de I993. Il en précise les origines et on remarque, ici comme ailleurs, combien les personnes rencontrées ne se rendent pas à la facilité de l'explication par le seul grossissement de la rivière. Il précise la manière dont l'eau est arrivée et exonère partiellement l'Yzeron de l'inondation. C'est moins la «faute» de la rivière que celle du ruissellement important sur la route départementale et sur le pont voisins. Une fois élucidées les origines de l'inondation, il dresse l'état des lieux de sa propriété et du quartier pendant la crue. Ces informations sont issues de son observation attentive et précise. Il donne ainsi à deux reprises la hauteur «manquant» à l'eau pour envahir sa propriété : vingt-sept centimètres. Une de ses moti- 
vations pourrait être de montrer la marge de manœuvre qu'il a conservée dans ces circonstances, pourtant dévastatrices pour nombre de propriétés des alentours. À l'état des lieux pendant la crue, s'ajoute la mention des différentes actions qu'il a engagées afin de contenir l'eau puis d'évacuer du mieux possible la boue entrée dans le garage. Sans transition, il passe au bilan de l'inondation : une collection de timbres perdue et des indemnisations correctes. Dans cette

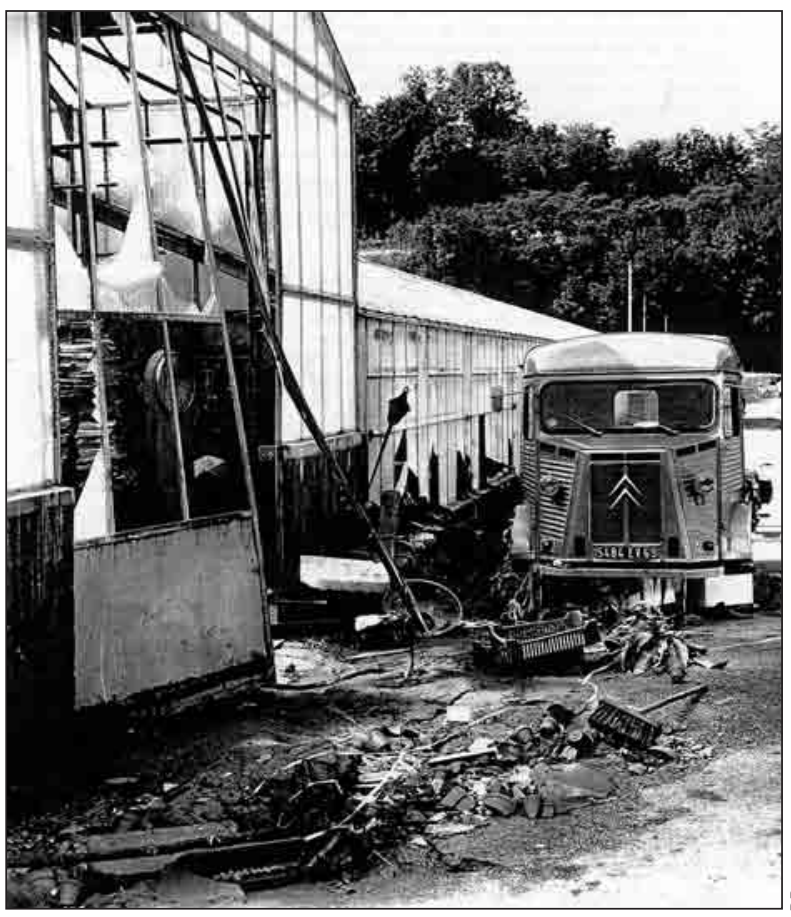

Les serres Morel après la crue du 11 août 1973.

succession d'événements et d'actions, point de place pour l'expression des émotions suscitées par l'envahissement des eaux. Ce récit circonstancié de l'inondation par une personne qui l'a vécue est particulièrement précis et «rassemblé». Il touche à différentes dimensions : on passe de l'extérieur de la maison à l'intérieur, de l'eau à la boue, des meubles aux timbres. Autre exemple, dans une composition plus mosaïque, une riveraine du Ravin donne les principales manifestations de la crue - un mètre d'eau dans les serres de son entreprise horticole, les «énormes dégâts », les modifications du ruisseau ainsi que les travaux qui ont été effectués, une qualification technique de l'orage et des éléments concernant sa propre situation professionnelle -, le tout avec des dates et des dimensions précises.

L'expérience de la crue distingue ceux qui ont vu de ceux à qui rien n'a été révélé. Si le glissement du sens quasi photographique au sens religieux du terme «révéler» est sans doute abusif, il n'en garde pas moins l'intérêt de situer cette expérience parmi celles qui font rupture avec «ce qui était ignoré de l'homme et incon- naissable par la raison $»^{6}$. En effet, la raison ne semble, en apparence seulement, d'aucune aide dans l'anticipation de la crue. «Il faut le voir pour le croire» semble être le leitmotiv, explicite ou implicite, des personnes «initiées », même si d'autres sens que la vue interviennent dans cette connaissance, rangée dans les croyances par l'emploi de cette expression familière.

\section{Un processus d'interprétation constamment renouvelé}

Tant en ce qui concerne l'Yzeron que le Ravin, des pluies continues ou un violent orage suscitent la vigilance des riverains, même si certains ne l'admettent pas facilement. En tout cas, les personnes rencontrées ne sont, en général, pas démunies devant la crue. Elles possèdent des éléments d'information, positionnent leur propre situation par rapport à ces éléments ou à d'autres situations qui leur sont connues. Nous ne pouvons faire comme si elles ne savaient rien des modalités d'occurrence des inondations. Les riverains ne restent, en effet, pas inertes devant les crues et les débordements de la rivière. Ils agissent aussi bien dans la dimension matérielle que dans la dimension idéelle. Les crues, leur origine, leur fréquence suscitent chez eux des tentatives d'interprétation visant à rendre raison de ces phénomènes. Ces tentatives mêlent souvent différents niveaux de réalité, allant de la prise en compte globale de l'environnement du ruisseau aux observations du régime des eaux, en passant par des soupçons plus ou moins fondés.

\section{Tentatives d'ordonnancement temporel}

Le régime torrentiel des deux cours d'eau étudiés est bien connu des riverains et souvent qualifié comme tel. Parfois, la durée des pluies intervient également et c'est leur volume accumulé qui semble seul expliquer le grossissement de la rivière. Cette montée rapide des eaux, combinée avec une descente de vitesse comparable, alimente souvent des spéculations de l'amont sur l'aval ou de l'aval sur l'amont du ruisseau. Dans la vallée du Ravin par exemple, beaucoup de riverains disent voir monter le niveau du cours d'eau en l'absence de pluies dans la vallée, puisque ce sont les orages du plateau de Rillieux qui sont largement responsables de l'alimentation en eau de la rivière. En fait, tout se passe comme si la description du régime de la rivière constituait, comme d'autres modalités, une forme d'appropriation de ces événements traumatiques et violents que sont crues et inondations.

La mémoire précise des dates de crues n'est pas l'apanage de tous les riverains rencontrés. Souvent,

6. Rey A. (dir.), (1998), Le Robert. Dictionnaire historique de la langue française, p. 3230 . 
la mémoire est plus confuse - en tous cas pour les épisodes anciens - même si, par ajustements successifs ou mobilisation d'événements personnels, les riverains situent globalement les inondations. Certaines personnes citent des dates précises, combinaison variable de jours, de mois et d'années. La répétition de ces dates «joue» même parfois comme un point de repère, peut-être comme une façon de rendre raison, de s'approprier un phénomène qui échappe, dans l'esprit de ces personnes, à tout autre cadre. En revanche, dans ce continuum de la vie au bord de la rivière, les dates précises de l'un ne sont pas les mêmes que celles de l'autre. Soulignant une fois de plus la rapidité de la montée et de la baisse des eaux, un riverain raconte un épisode dont la banalité n'est sans doute qu'apparente puisqu'il mentionne luimême l'importance qu'a pour lui cette date du 27 avril, jour où il s'était produit. D'autres jouent autrement avec le temps et les nombres qui l'incarnent. Certains évoquent une régularité de la rivière et de ses débordements. La périodicité prêtée aux crues est parfois cautionnée par une «autorité» scientifique, comme pour ce riverain qui se fie à ce que lui a dit un jour un technicien d'un service de l'État : «Votre maison n'est pas concernée, mais une catastrophe tous les quinze ans, c'est normal». Pour d'autres encore, c'est moins le continuum temporel qui offre un support aux débordements et permet de dégager le sens des événements, que les crues elles-mêmes qui déterminent des ruptures dans ce continuum d'ordinaire marqué par l'habitude.

\section{L'apparition d'embâcles urbains}

La plupart des personnes rencontrées s'interrogent sur les aménagements réalisés dans l'environnement immédiat de la rivière, voire sur le processus général d'urbanisation, et mettent en cause l'attitude des «gens» et, plus encore, des pouvoirs publics. Les protections individuelles que sont les murs édifiés le long du cours d'eau sont également évoquées, mais les mises en cause se font ici plus discrètes. Les accusations les plus fortes concernent des aménagements publics, ou considérés comme tels, jugés plus ou moins directement responsables. Pour le Ravin, la canalisation réalisée par la Communauté urbaine de Lyon et par la Direction départementale de l'Équipement, à la suite de la première inondation de I973, apparaît source de nouveaux maux et, pour certains, est directement responsable de la seconde inondation?. De fait, beaucoup de riverains conçoivent un «avant» et un «après » la canalisation qui a généré la cunette sinueuse et étroite où le Ravin s'écoule aujourd'hui. Pour certains, ce fut une «grave erreur» qu'ils paient par le classement de leur propriété en zone inondable. Pour d'autres, la configuration de la cunette n'est pas adaptée au lieu et les travaux ont été faits sans consultation et en dépit du bon sens.
Au-delà des institutions, c'est le partage du savoir entre «ingénieurs » et riverains que met en cause telle personne rencontrée : «[Avant le busage], on avait une voûte, là, qui faisait cinq mètres de large sur six mètres, je crois. C'était énorme. Il y avait bien une raison si les anciens avaient fait cette voûte». Ce faisant, il oublie que la voûte qu'il mentionne et qu'il attribue aux « anciens », a sans doute été bâtie par des ingénieurs et des techniciens et assimile par là même le passé aux seuls savoirs populaires. Du côté de l'Yzeron, le schéma est similaire, même si la nature des aménagements mis en cause diffère. Il s'agit ici des travaux de déviation d'une route départementale, le $C D$ 42. Les échos de ces travaux sont très clairement perçus dans les lotissements situés à proximité. Certains replacent les choix effectués - la circulation contre la préservation du bon écoulement des eaux dans la sphère politique. Au-delà parfois d'aménagements ciblés, la mise en cause est plus générale et concerne les pouvoirs publics désignés sans distinction par un «ils » impersonnel. Ils ont créé le risque avec les grandes infrastructures du secteur - la bretelle d'autoroute d'accès au contournement autoroutier de Lyon ou la ligne TGV Paris-Lyon. Cette globalisation des responsables conduit parfois à une politisation des rapports entre Administration et administrés, notamment en regard du rôle de l'État quant à la sécurité des personnes et des biens. Et, dans les cas évoqués, tout se passe comme si l'Administration créait, sinon la totalité, du moins une partie du risque.

L'urbanisation est surtout mobilisée comme un élément d'explication des crues par les riverains de l'Yzeron. Le fond de vallée y est, en effet, beaucoup plus occupé que celui du Ravin et les tailles des bassins versants ne sont guère comparables. Tel riverain explique les inondations actuelles et leur importance par l'urbanisation et les nombreux remblaiements de terrain auxquels se sont livrés les propriétaires en amont. Tel autre, en plus des eaux de ruissellement direct, rappelle celles qui, selon lui, sont captées par les réseaux d'assainissement et qui finissent également à la rivière. Souvent, dans les secteurs très urbanisés des communes en aval de l'Yzeron, les mises en cause se font plus précises quant à la densification du fond de vallée et à l'occupation de grands tènements industriels ou commerciaux autrefois dévolus à l'expansion des eaux. En revanche, pour des personnes plus éloignées de ce secteur, l'implantation d'un hypermarché sur une surface qui servait auparavant de champ d'expansion à la rivière ne peut être seule responsable des débordements qu'elles ont connus. Pour le Ravin, le phéno-

7. L'inondation de I973 avait eu pour conséquence la canalisation partielle du ruisseau, tandis que celle de I979 causée, semble-t-il, par des embâcles créés par la dégradation de la canalisation, a entraîné la réfection de cette dernière et le recalibrage de certains passages souterrains. 
mène d'urbanisation est moins immédiatement repérable car situé sur le plateau de Rillieux ${ }^{8}$, en amont de la source du cours d'eau, dans une configuration sociospatiale nettement différenciée9.

Quoi qu'il en soit, lorsque les riverains mettent en cause des aménagements publics ou le développement de l'urbanisation qui ont transformé les modalités d'alimentation et d'écoulement des cours d'eau, ce sont les administrations décentralisées et/ou les collectivités publiques qui sont interpellées. Même si la contradiction n'est pas souvent explicitée, ces opérations et les attitudes qu'elles dénotent posent problème en regard des interdictions faites aux riverains d'aménager des protections - murs, remblais pour l'essentiel. Telle riveraine met parfaitement au jour cette contradiction, en comparant l'accusation d'illégalité frappant ses cabanes à volailles à l'installation d'un container à verres par la municipalité de sa commune au bord du ruisseau. Les riverains répercutent donc ainsi, d'une certaine manière, les propos que les techniciens et les gestionnaires du risque opposent à leurs aménagements, et notamment à ceux qu'ils édifient pour se protéger.

Cette mise en parallèle des deux discours habitant et technicien n'est pas destinée à les neutraliser mutuellement. Elle souligne les incertitudes qui sont le lot des riverains quant aux modalités d'application des PPR et à ce qu'ils peuvent faire ou pourraient se permettre de faire par rapport à ce qu'autorise l'Administration. Qu'est-il possible de réaliser sur leur terrain désormais? Les dispositions du PPR remettent-elles en cause les constructions existantes et la manière dont elles sont agencées ? Qui prendrait en charge d'éventuels travaux de «mise aux normes» d'installations électriques ou autres? Autant d'interrogations entendues au cours de nos entretiens et de nos rencontres au bord des ruisseaux. Or, ces interrogations alimentent le questionnement sans fin que fait naître l'éventualité du danger d'inondation.

Certains de nos interlocuteurs ne tiennent même pas pour acquis un des éléments qui revient constamment dans les discours sur les inondations, à savoir l'augmentation croissante du nombre de crues. Pourtant tout le travail d'interprétation auquel se livrent les habitants rencontrés vise bien en général à rendre raison de ce fait rarement questionné ${ }^{10}$. Mais pour tel couple riverain de l'Yzeron depuis plus de quarantecinq ans, l'augmentation du nombre des crues ne va pas de soi. Ils jugent que le fait majeur réside dans l'augmentation de la population riveraine et donc des personnes potentiellement exposées au danger d'inondation. Pour ce qui les concerne directement, ils ne constatent pas d'augmentation particulière du nombre de crues en près de cinquante ans de résidence, tout en introduisant un rapport numérique entre cette durée de résidence et le nombre de crues réellement «inquiétantes $»^{\mathrm{II}}$. Sans être explicitement calculé, ce rapport légitime bien la longue cohabitation du couple avec la rivière. De même, un autre riverain, s'il ne cache pas qu'il surveille le cours d'eau, souligne la dimension socio-démographique de la construction du problème des crues en regard du nombre de personnes touchées. Peut-être sous-entend-il également ce changement dans les peuplements et les structures urbaines, souligné par quelques-uns.

\section{Le soupçon comme explication manquante}

Le danger potentiel d'inondation génère donc une activité d'interprétation importante, dont les fruits apparaissent moins comme des explications définitives que comme des étapes d'un processus sans cesse en mouvement. Au sein des tentatives d'interprétation que nous venons d'inventorier ou dans d'autres phases de l'entretien, les personnes rencontrées laissent souvent place au doute ou encore au soupçon. Élément moteur de ce processus, le soupçon apparaît comme un moyen à la fois de combler des vides d'interprétation et d'engendrer de nouvelles questions et perspectives d'interrogation. En effet, d'autres chercheurs ont constaté, y compris à d'autres échelles, que «les incertitudes qui entourent [les situations de danger potentiel] favorisent l'élaboration informelle, et parfois sauvage, d'hypothèses non encore vérifiées et souvent non immédiatement vérifiables » (Callon M. et alii, 200I, p. 42). Il est de fait difficile de circonscrire le champ ouvert par l'éventualité d'une crue et, plus encore, de clore les interrogations engendrées par le risque d'inondation et les peurs qu'il procure. Si les riverains ne sont pas à proprement parler démunis, il n'en reste pas moins qu'il manque parfois une pièce dans la mécanique bien rodée de leurs discours. Et ceci laisse alors place aux spéculations et, plus précisément, aux soupçons.

Du côté de l'Yzeron, les soupçons des riverains portent fréquemment sur l'éventuelle ouverture du barrage de la commune d'Yzeron, proche de la source. L'une des personnes rencontrées se lance dans une explication rationnelle rendant compte des difficultés d'écoulement de l'eau. Après avoir évoqué

8. Le PPR du Ravin était, au moment de sa publication à la fin de l'année I998, le seul à prendre en compte la totalité du bassin versant en définissant des zones d'aggravation du risque situées sur les balmes et sur les plateaux en amont.

9. Les grands ensembles de logements sociaux sont situés sur le plateau de Rillieux, tandis que la vallée du Ravin, en aval, est plutôt composée d'un tissu pavillonnaire.

Io. Nous ne retiendrons ici que le cas de l'Yzeron puisque celui du Ravin, en l'absence de crues majeures, ne correspond pas à ce cas de figure même s'il nous permet de remarquer qu'une éventuelle accélération des phénomènes de remplissage de la cunette ou des débordements ne fait l'objet d'aucune remarque.

II. Il utilise ici une procédure dont nous avons déjà parlé plus haut visant à inscrire les crues dans une régularité qui permet de les appréhender au mieux en gommant leur dimension traumatique. 
les travaux du CD 42, il montre comment le Rhône fait barrage en aval mais, sans doute toujours insatisfait de son explication, il éprouve le besoin de mobiliser autre chose : «ils auraient ouvert les portes du barrage de l'Yzeron à ce moment-là ». Il ne dispose manifestement d'aucun élément prouvant cette ouverture, mais cette dernière fait indéniablement partie d'un contexte conjoncturel. Envisager l'ouverture du barrage sert donc ici de chaînon manquant. D'autres fois, cette éventualité constitue à elle seule l'explication. Un autre riverain répète à trois reprises $q^{\prime}{ }^{\prime} \ll i l$ ne pleuvait plus et le niveau de l'eau est [pourtant] monté », comme s'il en était encore incrédule. Le lâcher du barrage peut seul expliquer, à ses yeux, la soudaineté de la montée des eaux. Il est d'ailleurs logique, si l'on veut bien admettre que les personnes qui s'en occupent avaient effectivement prévu la crue. Le soupçon est ainsi renforcé de la «rationalité » prêtée aux gestionnaires du barrage. Pour d'autres, le soupçon est en quelque sorte inversé et le barrage est, au contraire, crédité d'un rôle positif sur les crues, du fait d'une meilleure régulation de ses lâchers. Du coup, comme la gestion de la retenue d'eaux ne serait pour rien dans la montée des eaux, la recherche d'explications se poursuit avec un indice obtenu lors de la construction de la station-service de l'hypermarché, à savoir la présence de galeries

\section{RÉFÉRENCES}

Callon M., Lascoumes P., Barthe Y., (200I), Agir dans un monde incertain. Essai sur la démocratie technique, Le Seuil.

Coanus Th., (I992), «La thématique contemporaine du risque : entre demande sociale et recherche scientifique», in Le risque en montagne. Les réalités et les images, Éditions du CTHS, pp. I3-I9.

Dauphiné A., (200I), Risques et catastrophes. Observer, spatialiser, comprendre, gérer, Armand Colin.

Douglas M., (200I) (Ire édition I967), De la souillure. Essai sur les notions de pollution et de tabou, La Découverte, coll. Sciences humaines et sociales.

Dubois-Maury J., (200I), Les risques naturels : quelles réponses?, La Documentation française, collection Problèmes politiques et sociaux, Dossiers d'actualité mondiale, $n^{\circ} 863$.

Duchêne F., Morel Journel Ch., Coanus Th., Martinais E., (2003), La «culture du risque» en question(s), Éditions de l'Aube, à paraître. anciennes, datant de l'époque romaine, et qui conduiraient de l'eau souterraine à la rivière.

Tout montre que les crues, vécues ou imaginées, passées ou potentielles, génèrent chez les riverains des discours complexes, façonnés par des interprétations qui n'apparaissent que rarement figées. Ces interprétations semblent, au contraire, suffisamment réactives et mobiles pour intégrer sans cesse de nouveaux éléments, d'ordre technique et/ou rhétorique, à caractère collectif et/ou individuel. Elles s'alimentent aux incertitudes multiples qui caractérisent la situation des riverains, ainsi qu'à la peur engendrée par l'éventualité de l'inondation. Ainsi le risque que la crue sous-tend agit bien comme une représentation (Coanus Th., I992). Pour le dire autrement, et loin de cette division artificielle qui distingue aléa et vulnérabilité, «avec cette caractéristique que rien n'étant en soi un risque, tout peut en être un », tout dépend de la façon dont on analyse le danger, dont on considère l'événement (Ewald F., I986, p. I9), que l'on soit gestionnaire du risque ou que l'on soit riverain. Et, pour les riverains, les tentatives fécondes d'interprétation sont d'abord et avant tout des éléments pertinents pour continuer à vivre au bord de ces cours d'eau.

\section{François Duchêne, Christelle Morel Journel}

Duchêne F., Morel Journel Ch., (2000), «Riverains de cours d'eau et gestionnaires du risque, un dialogue impossible?», Géocarrefour (Revue de Géographie de Lyon), vol. $75, n^{\circ} 3$, pp. 22I-226.

Ewald F., (I986), L'État providence, Grasset et Fasquelle. Langumier J., (2002), Paroles et récits d'un territoire marqué par l'inondation. Enquête ethnologique auprès d'habitants de Cuxac d'Aude (11), mémoire de DEA de Sciences sociales sous la dir. de Françoise Zonabend, EHESS.

Reigner H., (2002), Les DDE et le politique. Quelle co-administration des territoires? L'Harmattan, collection Logiques politiques.

Zonabend F., (1989), La presqư̂le au nucléaire, Éditions Odile Jacob.

François Duchêne est docteur en géographie, chargé de recherches au Laboratoire RIVES - UMR-CNRS 5600, à l'École nationale des Travaux publics de l'État à Vaulx-en-Velin.

Christelle Morel Journel est docteur en géographie, maître de conférences à l'Université Jean Monnet de Saint-Étienne, et chercheur associé au Laboratoire RIVES.

$<$ duchene@entpe.fr $><$ ch.moreljournel@free.fr $>$ 